\title{
Epidemiology of SFTSV in Ticks collected from National Park in the ROK, 2015-2018
}

\author{
Yoon kyoung $\mathrm{Cho}^{2}$, jun gu kang ${ }^{2}$, young sun $\mathrm{jo}^{2}$, Sun-Woo $\mathrm{Han}^{2}$, Weon-Hwa Jheong ${ }^{1}$, Hye- \\ Sung Jeong ${ }^{1}$, Joon-seok Chae ${ }^{2}$ \\ ${ }^{1}$ Environmental Health Research Department, National Institute of Environmental Research, Hwangyeong-ro 42, Seo-gu, Incheon, 22689, Republic of \\ Korea., Incheon, Korea (the Republic of), ${ }^{2}$ Laboratoy of Veterinary Internal Medicine, BK21 PLUS program for Creative Veterinary Science Research, \\ Research Institute for Veterinary Science and College of Veterinary Medicine, Seoul National University, Seoul 08826, The Republic of Korea;, Seoul, \\ Korea (the Republic of)
}

\section{Objective}

To survey the distribution of Ixodid tick and infection of severe fever with thrombocytopenia syndrome virus (SFTSV) in natural environments from Deogyusan national parks in Korea.

\section{Introduction}

Severe fever with thrombocytopenia syndrome (SFTS) is an emerging viral disease in East-Asian countries, including China, Japan, and the Republic of Korea (ROK). The causative agent of SFTS is the SFTS virus (SFTSV) transmitted by hematophagous ticks.

\section{Methods}

To investigate the prevalence of SFTSV in the ROK, a total of 4,223 ticks were collected by flagging from Deogyusan National Park from 2015 to 2018. One-step reverse transcription-polymerase chain reaction (RT-PCR) and nested PCR were used to detect SFTSV-specific gene fragment from each ticks. The sequence data were analyzed using Chromas and aligned using CLUSTAL X. The phylogenetic analysis was constructed using the neighbor-joining method in MEGA7.

\section{Results}

Of the collected adult and nymph ticks, Haemaphysalis longicornis $(3611,85.5 \%)$ were the most abundant, followed by $H$. flava (502,11.88\%), Ixodes nipponensis $(109,2.5 \%)$, and Ixodes ovatus $(1,0.02 \%)$. The infection rate of SFTSV in total ticks was $5.8 \%$ $(245 / 4,223)$, and the infection rate by year was 3.69\% in 2015, 7.97\% in 2016, 5.08\% in 2017 and 4.68\% in 2018. The infection rates of SFTSV were getting decreased each year in Deogyusan National Park. In addition, infection rate was higher in spring and summer of each season. Phylogenetic analysis was performed and SFTSV sequences obtained in this study were included in Korean/Japanese SFTSV clade.

\section{Conclusions}

In conclusion, we confirmed the sequence of two clades, and it is thought that the epidemiological investigation of SFTSV is necessary through further studies. 